\title{
Caterpillar structures in single-wire Z-pinch experiments
}

\author{
M. D. Johnston, Y. Y. Lau, ${ }^{\text {a) }}$ R. M. Gilgenbach, T. S. Strickler, M. C. Jones, M. E. Cuneo, ${ }^{\text {b) }}$ \\ and T. A. Mehlhorn ${ }^{\text {b) }}$ \\ Intense Energy Beam Interaction Laboratory, Department of Nuclear Engineering and Radiological \\ Sciences, University of Michigan, Ann Arbor, Michigan 48109-2104
}

(Received 9 May 2003; accepted 15 October 2003)

\begin{abstract}
A series of experiments have been performed on single-wire $\mathrm{Z}$ pinches $(1-2 \mathrm{kA}, 20 \mathrm{kV}$, pulse length $500 \mathrm{~ns} ; \mathrm{Al}, \mathrm{Ag}, \mathrm{W}$, or $\mathrm{Cu}$ wire of diameter 7.5-50 $\mu \mathrm{m}$, length $2.5 \mathrm{~cm}$ ). Excimer laser absorption photographs show expansion of metallic plasmas on a time scale of order $100 \mathrm{~ns}$. The edge of this plasma plume begins to develop structures resembling a caterpillar only after the current pulse reaches its peak value. The growth of these caterpillar structures is shown to be consistent with the Rayleigh-Taylor instability of the decelerating plasma plume front at the later stage of the current pulse. (C) 2003 American Institute of Physics. [DOI: 10.1063/1.1632535]
\end{abstract}

Wire array $\mathrm{Z}$ pinches and the Sandia Z-accelerator have produced the most intense $\mathrm{X}$-ray pulses in the world ${ }^{1-5}$ with applications to radiation effects, ${ }^{1,3}$ inertial confinement fusion, ${ }^{2,4,5}$ and radiation sciences. These 100-200 terrawatt $\mathrm{x}$-ray pulses are generated in an array of 200-300 wires driven by multi-MJ, multi-MA pulsed power sources, with $\mathrm{x}$-ray energy yields in the MJ range. In these experiments, intense hydromagnetic activities have been observed. These experiments have been interpreted with magnetohydrodynamic simulations and models ${ }^{6-8}$ assumed to be seeded by random density perturbations of unspecified origin.

We have undertaken a modest experimental effort to characterize the gas/plasma of a single-wire $\mathrm{Z}$ pinch. The wire, made of $\mathrm{Al}, \mathrm{Ag}, \mathrm{W}$, or $\mathrm{Cu}$, of diameter ranging from 7.5 to $50 \mu \mathrm{m}$ and length $2.5 \mathrm{~cm}$, is subjected to a voltage and current pulse $(20 \mathrm{kV}, 2 \mathrm{kA})$ with the current rise time of order 200 ns. Optical diagnostics include emission spectroscopy with gated image-intensified CCD and resonant laser absorption. As in other recent work, ${ }^{9-12}$ these experiments are performed at $\sim 10 \mathrm{~A} /$ wire/ns, relevant to the conditions at early times during the first $50 \mathrm{~ns}$ of the current pulse in the MA Z-accelerator ( $\sim 30 \mathrm{~A} /$ wire/ns). This is during the socalled current prepulse, which sets up initial conditions for the main accelerating current pulse.

Our experiments reveal a puzzling phenomenon. Boundary perturbations resembling a caterpillar are developed at the outer edge of the still-expanding plasma plume [cf. Fig. 3]. The most unusual aspect of these structures is that they first appear only after the current reaches the peak value, and their growth rate is relatively mild. In virtually all previous wire Z-pinch experiments with high current pulses $(>100$ $\mathrm{kA} / 100 \mathrm{~ns}$ ), axial striations and radial jets were observed from the individual wire. ${ }^{7,13-16}$ The difference in these axial perturbations is that they grow violently, most pronounced during the current rise. They have mostly been attributed to the $m=0$ (sausage-like) instability or to the thermal instability, both in simulations and in speculations on their appearance. Moreover, unlike the caterpillar structures shown in

\footnotetext{
${ }^{a)}$ Electronic mail: yylau@umich.edu

${ }^{b)}$ Sandia National Laboratories, Albuquerque, NM 87185-1193.
}

this letter, these striations almost always develop rapidly, originating from the immediate vicinity of the metallic wire, and extending to the core. The initial condition of the wire is therefore of prime importance. In previous experiments with lower current pulses, ${ }^{9-12}$ with parameters similar to our experiments $(\sim 1.5 \mathrm{kA} / 500 \mathrm{~ns})$, axial structures with very fine scales were observed, extending to the wire core. In contrast, the caterpillar structures observed in our experiments always start late in the current pulse and develop from the plasma edge, and they do not seem to fit into previous observations. We interpret their occurrence in terms of Rayleigh-Taylor ( $\mathrm{R}-\mathrm{T}$ ) instability. Our analysis is qualitative, and is guided by the experimental data.

The single-wire Z-pinch experimental configuration is depicted in Fig. 1(a). A bank of six capacitors ( $2.5 \mathrm{nF}$ each) is charged to $17.5 \mathrm{kV}$, then discharged by a triggered spark-gap switch through a metal wire (typically $25 \mu \mathrm{m}$ Al) inside a turbomolecular-pumped vacuum chamber $\left(\sim 10^{-5}-10^{-6}\right.$ Torr). Voltage and current are monitored at the points shown in Fig. 1(a). Optical diagnostics are shown schematically in Fig. 1(b). Resonance-absorption photography of the wire plasma dynamics is performed utilizing either the $20 \mathrm{~ns}$ pulse from a $\mathrm{XeCl}$ laser $(308 \mathrm{~nm})$ or a $\mathrm{XeCl}-$ pumped dye laser. The $308 \mathrm{~nm}$ excimer laser line overlaps the $308 \mathrm{~nm}$ absorption line of aluminum neutral atoms. The spatial resolution of the system is $13 \mu \mathrm{m}$. Photos are taken with fast film and digitized. By adjusting the delay time of the laser relative to the current in the wire-plasma discharges, the temporal evolution of the plasma dynamics is investigated. The sensitivity range of resonance absorption diagnostics is approximately $10^{13} \mathrm{~cm}^{-2}$ line density (for $\mathrm{Al}$ ), ${ }^{17}$ orders of magnitude more sensitive than the diagnostics used in previous Z-pinch experiments. Presumably, it is this greatly improved sensitivity that allows the velocity measurements at the plasma edge, eventually leading to the interpretation of the caterpillar structures given in this letter.

The voltage and current measurements for the discharge of a $25-\mu \mathrm{m}$-diameter $\mathrm{Al}$ wire are presented in Fig. 2. The first $60 \mathrm{~ns}$ is a resistive phase of wire heating. During this phase, impurities (primarily hydrocarbons from oil used in wire manufacture) are released from the wire. Spectroscopic 

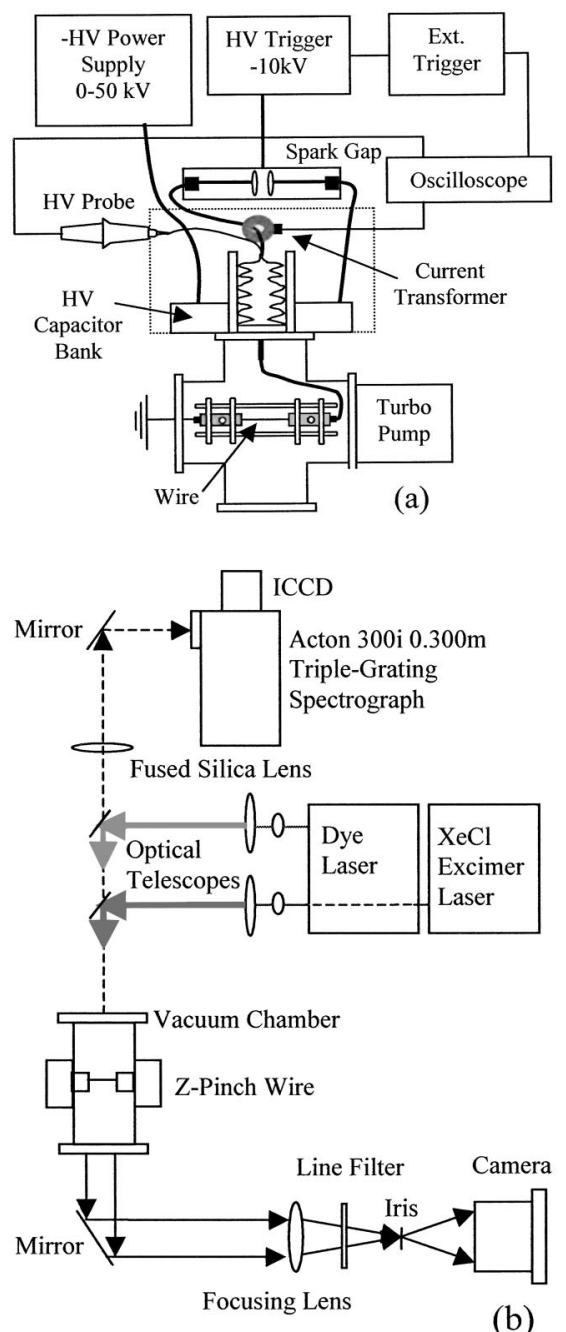

(b)

FIG. 1. (a) Experimental configuration of single-wire Z pinch and (b) Schematic illustration of optical diagnostics.

measurements (to be reported in a separate article) show that these impurities emit strong plasma line radiation (C II) before significant metal (Al) plasma emission is observed. The voltage and current traces show a discontinuity at about 60 ns; after this time, the resultant plasma discharge becomes primarily inductive, as shown by the $90^{\circ}$ phase lag between voltage and current. The current reaches its peak at about $250 \mathrm{~ns}$, (zero crossing of voltage). The current reverses sign at $500 \mathrm{~ns}$ (voltage maximum).

Figure 3 shows a sequence of $308 \mathrm{~nm}$ excimer laser

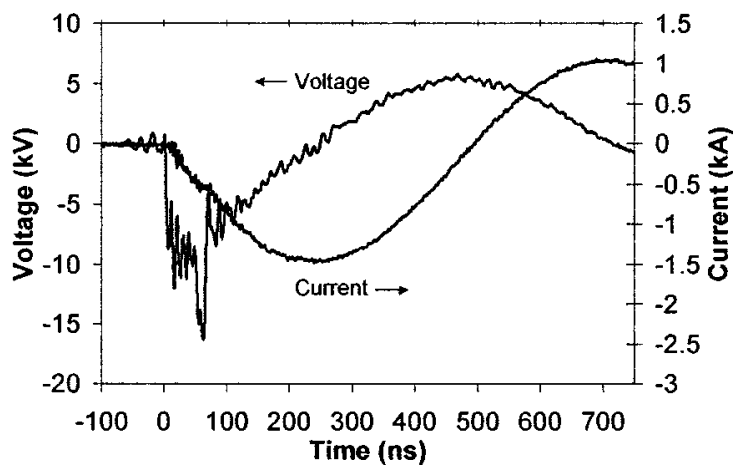

FIG. 2. Voltage and current measurements for the discharge of a $25 \mu \mathrm{m} \mathrm{Al}$ wire.

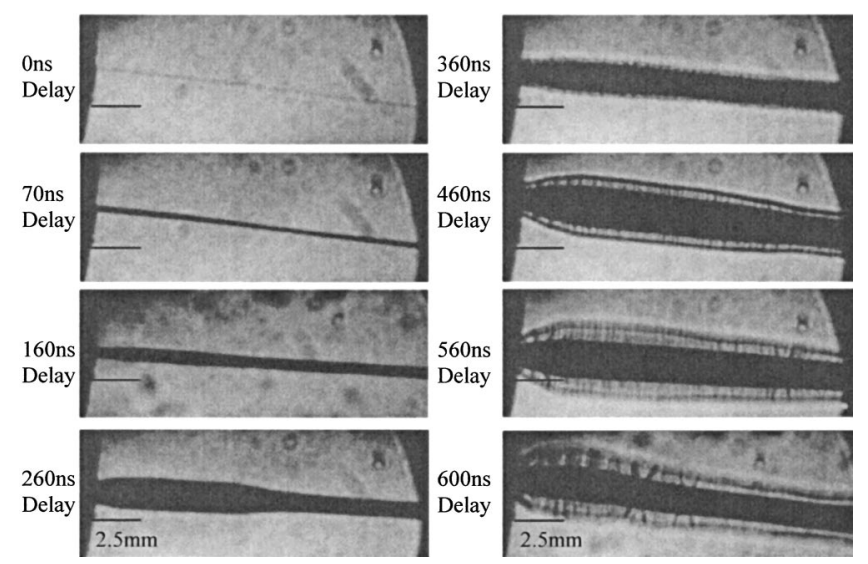

FIG. 3. Sequence of $\mathrm{XeCl}$ laser ( $308 \mathrm{~nm}, 20 \mathrm{~ns}$ ) absorption photographs of wire plasma discharge as a function of time.

absorption photographs as a function of time during the wire plasma discharge. It can be seen that the diameter of the dense aluminum plasma core expands rapidly with time as the current increases, until $260 \mathrm{~ns}$. In the region of falling current after the peak (360 ns photo), the core expansion slows down, and irregularities begin to be seeded along the length of the plasma boundary. Outside the opaque core region, one observes a corona sheath (starting at $360 \mathrm{~ns}$ ) with the evolving instability showing radial fingers. After $360 \mathrm{~ns}$, the core boundary begins to lose its integrity, as shown by the density striations, which become more visible and assume the caterpillar structure between 460 and 600 ns. A sharp edge (at $460 \mathrm{~ns}$ ) is presumably a limb-darkened feature due to chordal attenuation through the edge of an expanding cylindrical corona. A plot of the measured density striations' inner and outer radii versus time is shown in Fig. 4(a); definitions of these radii are shown in Fig. 4(b). Also shown in Fig. 4(a) is the mean radius, which we take to be the boundary of the unperturbed plume front. Note that the plume front's expansion velocity decreases beyond $300 \mathrm{~ns}$.

The caterpillar structures show up only during the later time, after the current reaches its peak value. They show up for wires of different materials and sizes. Their ubiquity and progressive growth suggest that they are caused by a hydromagnetic instability. Among the well-known hydromagnetic instabilities, the $m=0$ sausage mode driven by the current is ruled out because the growth does not occur at the peak current, nor during the current's rise time. Figure 3 shows no evidence of the $m=1$ kink mode. Kelvin-Helmholtz instability is unlikely either because of the apparent lack of multivelocity streams at the interface. This leaves the $\mathrm{R}-\mathrm{T}$ instability.

We argue, subsequently, that the $\mathrm{R}-\mathrm{T}$ instability qualitatively matches the observed evolution of the caterpillar structures. During the rise time of the current, one envisions that the metallic plasma plume front is accelerated radially outward by the resistive energy deposition. In this phase, the direction of acceleration, in the lab frame, is from the heavy fluid (metallic plasma) into a light fluid (vacuum, or lowdensity plasma) and the interface is $\mathrm{R}-\mathrm{T}$ stable. After the current reaches its peak value, the metallic plasma plume slows down. Thus, the direction of acceleration in this latter phase, in the lab frame, is from the light fluid (vacuum, or 

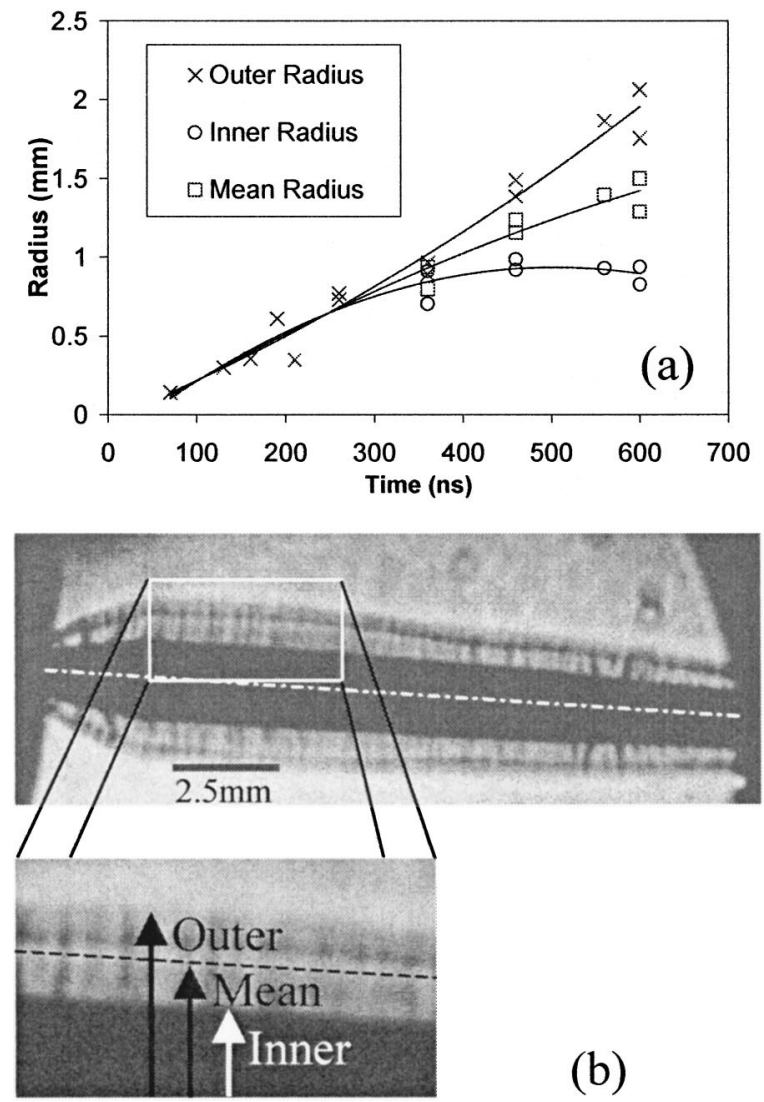

FIG. 4. (a) Experimental data of the measured inner radius (circles), outer radius $(\mathrm{X})$, and mean value (squares) versus time for the wire plasma discharge (25 $\mu \mathrm{m} \mathrm{Al}$ wire). Data were taken at $4.5 \mathrm{~mm}$ from the anode. (b) Definitions of the radii, using the enlarged $t=560 \mathrm{~ns}$ frame of Fig. 3.

low-density plasma) to the heavy fluid (metallic plasma) and the interface becomes $\mathrm{R}-\mathrm{T}$ unstable.

Referring to Fig. 4(a), we then interpret the progressive separation between the outer and inner diameter (i.e., the growing caterpillar "legs") simply as the R-T growth of the unstable boundary ripple of the slowing-down plasma plume front. Lacking experimental evidence to the contrary, we postulate that the observed axial wavelength $\left(\lambda=2 \pi / k_{m}\right)$, on the order of $0.00025 \mathrm{~m}$ from the density striations in Fig. 3 , corresponds to the axial wavenumber $\left(k_{m}\right)$ that yields the fastest growth rate $\left(\gamma_{m}\right)$ in the R-T instability. To show that this interpretation is consistent with the caterpillar growth, let us write, upon using the scaling for the R-T instability,

$$
\gamma_{m}=p \sqrt{k_{m} g},
$$

where $g$ is the acceleration of the interface, which is taken to be positive if the acceleration (in the lab frame) is from the lighter fluid to the heavy fluid, and $p$ is a constant.

The value of $p$ is on the order of, but less than, unity; we take $p=0.75$ using the following arguments. It is easy to show that a dispersion relation of the form $\gamma^{2}=g k-A k^{\beta}$ yields the maximum growth rate $\gamma=\gamma_{m}$ at $k=k_{m}$ given by Eq. (1). The values of $p$ are $0.707,0.816$, and 0.866 , respectively, for $\beta=2,3$, and 4 , regardless of the value of the constant $A(A>0)$. Note that this range of $p$ is rather narrow, and that $\beta=2,3,4$ may represent stabilizing mechanisms due, respectively, to thermal effects, surface tension, and viscosity. Thus, $p$ is expected to be on the order of, but less than, unity, regardless of the stabilizing mechanism. We remark that even with the "fire-polishing" effect, the dispersion relation $\gamma=(g k)^{1 / 2}-k \nu_{A}$ would yield the maximum growth rate given by Eq. (1) with $p=0.5$, regardless of $\nu_{A}(>0)$, which is the velocity measuring the ablation rate. ${ }^{18}$

We estimate $g=5.7 \times 10^{9} \mathrm{~m} / \mathrm{s}^{2}$, by comparing the deceleration of the plume front velocity from 300 to $600 \mathrm{~ns}$ [Fig. 4(a)]. With $\lambda=0.00025 \mathrm{~m}$, we have $k_{m}=2 \pi / \lambda=2.5$ $\times 10^{4} \mathrm{~m}^{-1}$. Using these values of $p, g$, and $k_{m}$ in Eq. (1), we obtain $\gamma_{m}=0.9 \times 10^{7} \mathrm{~s}^{-1}$. Thus, in $300 \mathrm{~ns}$, the amplitude of the $\mathrm{R}-\mathrm{T}$ instability increases by a factor of $e^{2.7}=15$, which is on the order of magnitude of the growth in the caterpillar size from 300 to 600 ns, as shown in Fig. 4(a). Note that this represents a mild $\mathrm{R}-\mathrm{T}$ growth.

We may even estimate the initial boundary perturbation $\left(s_{i n}\right)$ that seeds the R-T growth. Roughly, the boundary perturbation $[s(t)]$ at time $t$ is related to the initial perturbation (at time $\left.t_{\text {in }}\right)$ by $s(t) \approx s_{\text {in }} \exp \left[\gamma_{m}\left(t-t_{\text {in }}\right)\right]$. From Fig. 4(b), we take $t=560 \mathrm{~ns}$, at which $s=470 \mu \mathrm{m}$ (= separation between the outer and mean radius). Assuming that $\mathrm{R}-\mathrm{T}$ instability begins at $t_{\text {in }}=260 \mathrm{~ns}$ (Fig. 2), and using $\gamma_{m}=0.9 \times 10^{7} \mathrm{~s}^{-1}$, we then obtain the initial perturbation at the onset of the $\mathrm{R}-\mathrm{T}$ instability to be $s_{\text {in }}=31 \mu \mathrm{m}$, which is on the order of, but larger than, the initial wire diameter.

This work was supported by U.S. DoE by contract number BE-6069 from the Sandia National Laboratories.

${ }^{1}$ C. A. Coverdale, C. Deeney, and M. R. Douglas, Phys. Rev. Lett. 88, 065001-1 (2002).

${ }^{2}$ M. E. Cuneo et al., Phys. Plasmas 8, 2257 (2001).

${ }^{3}$ C. Deeney, C. A. Coverdale, and M. R. Douglas, Laser Part. Beams 19, 497 (2001).

${ }^{4}$ L. I. Rudakov, A. L. Velikovich, J. Davis, J. W. Thornhill, J. L. Giuliani, Jr., and C. Deeney, Phys. Rev. Lett. 84, 3326 (2000).

${ }^{5}$ M. E. Cuneo, R. A. Vesey, J. L. Porter, Jr., G. R. Bennett, D. L. Hanson, L. E. Ruggles, W. W. Simpson, G. C. Idzorek, W. A. Stygar, J. H. Hammer, J. J. Seamen, J. A. Torres, J. S. McGurn, and R. M. Green, Phys. Rev. Lett. 88, 215004-1 (2002).

${ }^{6}$ J. H. Hammer, M. Tabak, S. C. Wilks, J. O. Lindl, D. S. Bailey, P. W. Rambo, A. Toor, G. B. Zimmerman, and J. L. Porter, Jr., Phys. Plasmas 6, 2129 (1999).

${ }^{7}$ J. P. Chittenden, S. V. Lebedev, S. N. Bland, J. Ruiz-Camacho, F. N. Beg, and M. G. Haines, Laser Part. Beams 19, 323 (2001); Phys. Plasmas 8, 2305 (2001); Phys. Rev. E 61, 4370 (2000).

${ }^{8}$ M. P. Desjarlais and B. M. Marder, Phys. Plasmas 6, 2057 (1999).

${ }^{9}$ S. A. Pikuz, T. A. Shelekovenko, D. B. Sinars, J. B. Greenly, Y. S. Dimant, and D. A. Hammer, Phys. Rev. Lett. 83, 4313 (1999).

${ }^{10}$ D. B. Sinars, T. A. Shelekovenko, S. A. Pikuz, J. B. Greenly, and D. A. Hammer, Phys. Plasmas 7, 1555 (2000).

${ }^{11}$ P. U. Duselis and B. R. Kusse, Phys. Plasmas 10, 565 (2003).

${ }^{12}$ K. M. Chandler, D. A. Hammer, D. B. Sinars, S. A. Pikuz, and T. A. Shelkovenko, IEEE Trans. Plasma Sci. 30, 577 (2002).

${ }^{13}$ D. H. Kalantar and D. A. Hammer, Phys. Rev. Lett. 71, 3806 (1993).

${ }^{14}$ S. V. Lebedev, F. N. Beg, S. N. Bland, J. P. Chittenden, A. E. Dangor, M. G. Haines, S. A. Pikuz, and T. A. Shelkovenko, Phys. Rev. Lett. 85, 98 (2000).

${ }^{15}$ S. Y. Gus'kov, G. V. Ivanenkov, A. R. Mingaleev, S. A. Pikuz, T. A. Shelkovenko, and D. A. Hammer, JETP Lett. 67, 559 (1998); G. S. Sarkisov, B. S. Bauer, and J. S. DeGroot, ibid. 73, 69 (2001); G. V. Ivanenkov, A. R. Mingaleev, S. A. Pikuz, V. M. Romanova, T. A. Shelkovenko, W. Stepniewski, and D. A. Hammer, JETP 87, 663 (1998).

${ }^{16}$ S. A. Pikuz, T. A. Shelkovenko, A. R. Mingaleev, D. A. Hammer, and H. P. Neves, Phys. Plasmas 6, 4272 (1999).

${ }^{17}$ R. M. Gilgenbach, C. H. Ching, J. S. Lash, and R. A. Lindley, Phys. Plasmas 1, 1619 (1994).

${ }^{18}$ S. E. Bodner, Phys. Rev. Lett. 33, 761 (1974). 